Preprint typeset in JHEP style - HYPER VERSION

\title{
Lovelock-Lifshitz Black Holes
}

\author{
M. H. Dehghani \\ 1. Department of Physics, University of Waterloo, 200 University Avenue West, \\ Waterloo, Ontario, Canada, N2L $3 G 1$ \\ 2. Physics Department and Biruni Observatory, College of Sciences, \\ Shiraz University, Shiraz 71454, Iran \\ E-mail: dehghani@uwaterloo.ca, mhd@shirazu.ac.ir
}

\section{R. B. Mann}

Department of Physics, University of Waterloo, 200 University Avenue West, Waterloo, Ontario, Canada, N2L 3G1

E-Mail: rbmann@sciborg.uwaterloo.ca

ABSTRACT: In this paper, we investigate the existence of Lifshitz solutions in Lovelock gravity, both in vacuum and in the presence of a massive vector field. We show that the Lovelock terms can support the Lifshitz solution provided the constants of the theory are suitably chosen. We obtain an exact black hole solution with Lifshitz asymptotics of any scaling parameter $z$ in both Gauss-Bonnet and in pure 3rd order Lovelock gravity. If matter is added in the form of a massive vector field, we also show that Lifshitz solutions in Lovelock gravity exist; these can be regarded as corrections to Einstein gravity coupled to this form of matter. For this form of matter we numerically obtain a broad range of charged black hole solutions with Lifshitz asymptotics, for either sign of the cosmological constant. We find that these asymptotic Lifshitz solutions are more sensitive to corrections induced by Lovelock gravity than are their asymptotic AdS counterparts. We also consider the thermodynamics of the black hole solutions and show that the temperature of large black holes with curved horizons is proportional to $r_{0}^{z}$ where $z$ is the critical exponent; this relationship holds for black branes of any size. As is the case for asymptotic AdS black holes, we find that an extreme black hole exists only for the case of horizons with negative curvature. We also find that these Lovelock-Lifshitz black holes have no unstable phase, in contrast to the Lovelock-AdS case. We also present a class of rotating Lovelock-Lifshitz black holes with Ricci-flat horizons.

Keywords: Black Holes, Gauge-gravity correspondence, AdS-CFT correspondence. 


\section{Contents}

1. Introduction 1

2. Field equations 3

3. Lifshitz Solutions 5

3.1 Vacuum Solutions 5

3.2 Matter Solutions 6

4. Asymptotic Lifshitz Black holes 8

4.1 Series solutions near the horizon 8

4.2 Numeric Solutions 8

5. Thermodynamics of black holes 13

6. Rotating Lovelock-Lifshitz solutions

7. Concluding Remarks 19

8. Appendix 20

\section{Introduction}

Candidates for gravity duals of non-relativistic scale invariant theories have recently attracted a great deal of attention for several reasons. One is that some condensed matter systems realized in laboratories at their critical points are described by nonrelativistic conformal field theories. Furthermore, the AdS/CFT correspondence [1, 2] describing the duality between strongly coupled conformal field theory and gravity continues to find more applications in other branches of physics such as QCD quarkgluon plasmas [3], atomic physics, and condensed matter physics [4, 5, 6, 7, 8, 9]. This has led to a study of gravity-gauge duality in a much broader context than its original AdS/CFT formulation, extending to non-relativistic and Lifshitz field theories, with the aim of gaining more knowledge of the strong coupling behavior of these other physical systems. Non-relativistic conformal symmetry contains the scaling symmetry

$$
t \rightarrow \lambda^{z} t, \quad \mathbf{x} \rightarrow \lambda \mathbf{x}
$$


where $\mathrm{z}$ is the dynamical exponent. This transformation exhibits anisotropic scale invariant behavior. Actually, for condensed matter applications, one typically is interested in considering anisotropy between different spatial dimensions. For $z=1$ this scaling symmetry is the familiar conformal symmetry. Such a non-relativistic scale invariance (1.1) can be exhibited by either a Galilean-invariant theory or a Lifshitz-invariant theory. From a holographic point of view, this suggests the following (asymptotic) form for the spacetime metric

$$
d s^{2}=L^{2}\left(-r^{2 z} d t^{2}+\frac{d r^{2}}{r^{2}}+r^{2} d \mathbf{x}^{2}\right)
$$

that obeys the scale invariance

$$
t \rightarrow \lambda^{z} t, \quad r \rightarrow \lambda^{-1} r, \quad \mathbf{x} \rightarrow \lambda \mathbf{x}
$$

noted previously in a braneworld context 10].

A four-dimensional anisotropic scale invariant background using an action involving a two form and a three form field with a Chern-Simons coupling

$$
I=\int d^{4} x \sqrt{-g}\left(R-2 \Lambda-\frac{1}{4} F_{\alpha \beta} F^{\mu \nu}-\frac{1}{12} H_{\mu \nu \rho} H^{\mu \nu \rho}-\frac{C}{\sqrt{-g}} F \wedge B\right),
$$

can be engineered to yield solutions with this asymptotic behavior [11], where $H=$ $d B$ and $F=d A$. Such an action was argued to be rather generic in string theory, although no explicit brane realization or embedding into ten-dimensions was given. For these matter fields, lots of effort has been expended in extending this solution to the case of asymptotic Lifshitz solutions. One of the first analytic examples was reported in Ref. [12] for a sort of higher-dimensional dilaton gravity without restricting the value of the dynamical exponent $z$. An exact topological black hole solution with hyperbolic horizon which happens to be asymptotically Lifshitz with $z=2$ was found in [13; further solutions with $z=4$ and with spherical topology were subsequently obtained [14]. However in general such asymptotic Lifshitz black holes must be investigated numerically [13, 14, 15, 16]. Other possibilities for the matter needed to support such a background have been investigated [12]. Asymptotic Lifshitz solutions in the vacuum of higher-derivative gravity theories (with curvaturesquared terms in the action) have been investigated [17, 18, 19, 20]; the higher curvature terms with suitable coupling constant play the role of the desired matter.

Recently gravity theories including higher powers of the curvature, particularly curvature-cubed interactions, have attracted increased attention [21]. This is because, in the context of AdS/CFT correspondence, corrections from higher powers of the curvature must be considered on the gravity side of the correspondence in order to scrutinize CFTs with different values for their central charges. Amongst the cornucopia of higher-curvature gravity theories, Lovelock gravity theories play a 
special role in that the number of metric derivatives in any field equation is never larger than 2. Third-order Lovelock gravity is supersymmetric, and therefore one can define superconformal field theories via the AdS/CFT correspondence [22, 23]. Furthermore quasi-topological gravity including curvature-cubed interactions, while not supersymmetric, can be considered to be dual to some non-supersymmetric but conformal gauge theory in the limit of a large number of colours [24].

In this paper, we consider the existence of Lifshitz solutions in third order Lovelock gravity both in vacuum and in the presence of a massive vector field. Since the higher curvature terms appear to play the role of some kind of matter field, it is natural to ask whether they can support a Lifshitz solution in vacuum or not. We find that the answer is yes, albeit under restricted circumstances. We also search for asymptotic Lifshitz black holes in the presence of a massive vector field, whose action is given via a dualization of the action (1.4). The solutions we find - both analytically and numerically - can be regarded as higher-curvature modifications to those obtained from Einsteinian gravity coupled to matter [13, 14, 16].

The outline of our paper is as follows. We give a brief review of the field equations of third order Lovelock gravity in the presence of massive vector field in Sec. 2. In Sec. 3 we present the $(n+1)$-dimensional exact Lifshitz solutions in vacuum and in the presence of a massive vector field. In Sec. 14 we obtain the series solutions to the field equations near the horizon, while the series solutions at large $r$ will be given the Appendix. We then obtain numerical solutions to these equations. The thermodynamics of these Lovelock-Lifshitz black holes will be given in Sec. 5. Section 6 will be devoted to the rotating Lovelock-Lifshitz solutions. We finish our paper with some concluding remarks.

\section{Field equations}

The fundamental assumptions in standard general relativity are the requirements of general covariance and 2nd-order differential field equations for the metric. Based on the same principles, the Lovelock Lagrangian is a very general Lagrangian in classical gravity that produces second order field equations for the metric for arbitrary powers of the curvature [25]. The action of third order Lovelock gravity in the presence of an Abelian massive vector field $A^{\mu}$ may be written as

$$
I=\int d^{n+1} x \sqrt{-g}\left(-2 \Lambda+\mathcal{L}_{1}+\alpha_{2} \mathcal{L}_{2}+\alpha_{3} \mathcal{L}_{3}-\frac{1}{4} F_{\mu \nu} F^{\mu \nu}-\frac{1}{2} m^{2} A_{\mu} A^{\mu}\right)
$$

where $F_{\mu \nu}=\partial_{[\mu} A_{\nu]}, \Lambda$ is the cosmological constant, $\alpha_{2}$ and $\alpha_{3}$ are Gauss-Bonnet and third order Lovelock coefficients, $\mathcal{L}_{1}=R$ is the Einstein-Hilbert Lagrangian, $\mathcal{L}_{2}=R_{\mu \nu \gamma \delta} R^{\mu \nu \gamma \delta}-4 R_{\mu \nu} R^{\mu \nu}+R^{2}$ is the Gauss-Bonnet Lagrangian, and 


$$
\begin{aligned}
\mathcal{L}_{3}= & R^{3}+2 R^{\mu \nu \sigma \kappa} R_{\sigma \kappa \rho \tau} R^{\rho \tau}{ }_{\mu \nu}+8 R_{\sigma \rho}^{\mu \nu} R_{\nu \tau}^{\sigma \kappa} R_{\mu \kappa}^{\rho \tau}+24 R^{\mu \nu \sigma \kappa} R_{\sigma \kappa \nu \rho} R^{\rho}{ }_{\mu} \\
& +3 R R^{\mu \nu \sigma \kappa} R_{\sigma \kappa \mu \nu}+24 R^{\mu \nu \sigma \kappa} R_{\sigma \mu} R_{\kappa \nu}+16 R^{\mu \nu} R_{\nu \sigma} R^{\sigma}{ }_{\mu}-12 R R^{\mu \nu} R_{\mu \nu}
\end{aligned}
$$

is the third order Lovelock Lagrangian. We assume that the Gauss-Bonnet coefficient, which has the dimension of (length $)^{2}$, is positive as in the heterotic string theory [26].

In Lovelock gravity only terms with order less than $[(n+1) / 2]$ (where $[x]$ is the integer part of $x$ ) contribute to the field equations, the rest being total derivatives in the action. For 3rd-order Lovelock gravity we therefore consider $(n+1)$-dimensional spacetimes with $n \geq 6$ (though in situations where we set $\hat{\alpha}_{3}=0$ our solutions will be valid for $n \geq 4$ ). Varying the action with respect to the metric tensor $g_{\mu \nu}$ and gauge field $A_{\mu}$ the equations of gravitation and gauge fields are

$$
\begin{aligned}
& G_{\mu \nu}^{(1)}+\alpha_{2} G_{\mu \nu}^{(2)}+\alpha_{3} G_{\mu \nu}^{(3)}+\Lambda g_{\mu \nu}=T_{\mu \nu}, \\
& \partial_{\mu}\left(\sqrt{-g} F^{\mu \nu}\right)=m^{2} \sqrt{-g} A^{\nu}
\end{aligned}
$$

where

$$
T_{\mu \nu}=\frac{1}{2}\left(F^{\rho}{ }_{\mu} F_{\rho \nu}-\frac{1}{4} F_{\rho \sigma} F^{\rho \sigma} g_{\mu \nu}+m^{2}\left[A_{\mu} A_{\nu}-\frac{1}{2} A_{\lambda} A^{\lambda} g_{\mu \nu}\right]\right)
$$

is the energy-momentum tensor of gauge field, $G_{\mu \nu}^{(1)}$ is just the Einstein tensor, and $G_{\mu \nu}^{(2)}$ and $G_{\mu \nu}^{(3)}$ are given as:

$$
\begin{aligned}
& G_{\mu \nu}^{(2)}=2\left(-R_{\mu \sigma \kappa \tau} R^{\kappa \tau \sigma}{ }_{\nu}-2 R_{\mu \rho \nu \sigma} R^{\rho \sigma}-2 R_{\mu \sigma} R^{\sigma}{ }_{\nu}+R R_{\mu \nu}\right)-\frac{1}{2} \mathcal{L}_{2} g_{\mu \nu}, \\
& G_{\mu \nu}^{(3)}=-3\left(4 R^{\tau \rho \sigma \kappa} R_{\sigma \kappa \lambda \rho} R_{\nu \tau \mu}^{\lambda}-8 R^{\tau \rho}{ }_{\lambda \sigma} R^{\sigma \kappa}{ }_{\tau \mu} R_{\nu \rho \kappa}^{\lambda}+2 R_{\nu}^{\tau \sigma \kappa} R_{\sigma \kappa \lambda \rho} R_{\tau \mu}^{\lambda \rho}\right. \\
& -R^{\tau \rho \sigma \kappa} R_{\sigma \kappa \tau \rho} R_{\nu \mu}+8 R_{\nu \sigma \rho}^{\tau} R_{\tau \mu}^{\sigma \kappa} R_{\kappa}^{\rho}+8 R_{\nu \tau \kappa}^{\sigma} R_{\sigma \mu}^{\tau \rho} R_{\rho}^{\kappa} \\
& +4 R_{\nu}{ }^{\tau \sigma \kappa} R_{\sigma \kappa \mu \rho} R_{\tau}^{\rho}-4 R_{\nu}{ }^{\tau \sigma \kappa} R_{\sigma \kappa \tau \rho} R_{\mu}^{\rho}+4 R^{\tau \rho \sigma \kappa} R_{\sigma \kappa \tau \mu} R_{\nu \rho}+2 R R_{\nu}{ }^{\kappa \tau \rho} R_{\tau \rho \kappa \mu} \\
& +8 R_{\nu \mu \rho}^{\tau} R_{\sigma}^{\rho} R_{\tau}^{\sigma}-8 R_{\nu \tau \rho}^{\sigma} R_{\sigma}^{\tau} R_{\mu}^{\rho}-8 R_{\sigma \mu}^{\tau \rho} R_{\tau}^{\sigma} R_{\nu \rho}-4 R R_{\nu \mu \rho}^{\tau} R_{\tau}^{\rho} \\
& \left.+4 R^{\tau \rho} R_{\rho \tau} R_{\nu \mu}-8 R^{\tau}{ }_{\nu} R_{\tau \rho} R^{\rho}{ }_{\mu}+4 R R_{\nu \rho} R^{\rho}{ }_{\mu}-R^{2} R_{\nu \mu}\right)-\frac{1}{2} \mathcal{L}_{3} g_{\mu \nu} .
\end{aligned}
$$

The metric of an $(n+1)$-dimensional asymptotically Lifshitz static and radially symmetric spacetime may be written as:

$$
d s^{2}=-\frac{r^{2 z}}{l^{2 z}} f(r) d t^{2}+\frac{l^{2} d r^{2}}{r^{2} g(r)}+r^{2} d \Omega^{2}
$$

where the functions $f(r)$ and $g(r)$ must go to 1 as $r$ goes to infinity. In Eq. (2.5) $d \Omega^{2}$ is the metric of an $(n-1)$-dimensional hypersurface with constant curvature 
$(n-1)(n-2) k$ and volume $V_{n-1}$. We can write

$$
d \Omega^{2}=\left\{\begin{array}{cc}
d \theta_{1}^{2}+\sum_{i=2}^{n-1} \prod_{j=1}^{i-1} \sin ^{2} \theta_{j} d \theta_{i}^{2} & k=1 \\
d \theta_{1}^{2}+\sinh ^{2} \theta_{1}\left(d \theta_{2}^{2}+\sum_{i=3}^{n-1} \prod_{j=2}^{i-1} \sin ^{2} \theta_{j} d \theta_{i}^{2}\right) & k=-1 \\
\sum_{i=1}^{n-1} d \theta_{i}^{2} & k=0
\end{array}\right.
$$

though it should be noted that our solutions are valid whenever $d \Omega^{2}$ describes any Einstein space.

Using the ansatz

$$
A_{t}=q \frac{r^{z}}{l^{z}} h(r)
$$

for the gauge field and defining $\hat{\alpha}_{2} \equiv(n-2)(n-3) \alpha_{2}$ and $\hat{\alpha}_{3} \equiv(n-2) \ldots(n-5) \alpha_{3}$ for convenience, the field equations (2.3) and (2.4) reduce to the system

$$
\begin{aligned}
& 2 r^{2} h^{\prime \prime}-r\left[(\ln f)^{\prime}-(\ln g)^{\prime}\right]\left(r h^{\prime}+z\right)-2(n+z) r h^{\prime}+2(n-1) z=\frac{2 m^{2} l^{2}}{g} \\
& r^{4} l^{4}\left\{n(n-1) r^{2} g+(n-1) r^{3} g^{\prime}+2 \Lambda l^{2} r^{2}-(n-1)(n-2) k l^{2}\right\} \\
& +(n-1) \hat{\alpha}_{2} l^{2} r^{2}\left(k l^{2}-r^{2} g\right)\left\{n r^{2} g+2 r^{3} g^{\prime}-(n-4) k l^{2}\right\} \\
& +(n-1) \hat{\alpha}_{3}\left(k l^{2}-r^{2} g\right)^{2}\left\{n r^{2} g+3 r^{3} g^{\prime}-(n-6) k l^{2}\right\}=2 l^{6} r^{6} T_{t}^{t}, \\
& r^{4} l^{4}\left\{(n-1)(n-2+2 z) r^{2} g+(n-1) r^{3} g(\ln f)^{\prime}+2 \Lambda l^{2} r^{2}-(n-1)(n-2) k l^{2}\right\} \\
& +(n-1) \hat{\alpha}_{2} l^{2} r^{2}\left(k l^{2}-r^{2} g\right)\left\{(n-4+4 z) r^{2} g+2 r^{3} g(\ln f)^{\prime}-(n-4) k l^{2}\right\} \\
& +(n-1) \hat{\alpha}_{3}\left(k l^{2}-r^{2} g\right)^{2}\left\{(n-6+6 z) r^{2} g+3 r^{3} g(\ln f)^{\prime}-(n-6) k l^{2}\right\} \\
& \quad=2 l^{6} r^{6} T_{r}^{r},
\end{aligned}
$$

where prime denotes the derivative with respect to $r$ and $T_{t}^{t}$ and $T_{r}^{r}$ are

$$
\begin{aligned}
& T_{t}^{t}=-\frac{q^{2}}{4 l^{2} f}\left\{g\left(r h^{\prime}+z h\right)^{2}+m^{2} l^{2} h^{2}\right\}, \\
& T_{r}^{r}=-\frac{q^{2}}{4 l^{2} f}\left\{g\left(r h^{\prime}+z h\right)^{2}-m^{2} l^{2} h^{2}\right\} .
\end{aligned}
$$

\section{Lifshitz Solutions}

\subsection{Vacuum Solutions}

We first investigate the possibility of having $(n+1)$-dimensional Lifshitz solutions

$$
d s^{2}=-\frac{r^{2 z}}{l^{2 z}} d t^{2}+\frac{l^{2} d r^{2}}{r^{2}}+r^{2} \sum_{i=1}^{n-1} d \theta_{i}^{2},
$$


in Lovelock gravity in the absence of matter. In order to have an asymptotically Lifshitz solution in 3rd-order Lovelock gravity, the following constraints on the cosmological constant and third order Lovelock coefficient

$$
\Lambda=-\frac{n(n-1)}{6 l^{4}}\left(2 l^{2}-\hat{\alpha}_{2}\right), \quad \hat{\alpha}_{3}=-\frac{l^{2}}{3}\left(l^{2}-2 \hat{\alpha}_{2}\right) .
$$

hold for an arbitrary value of $z$, as is easily obtained via straightforward calculation.

Note that for $\alpha_{3}=0$ the constraints (3.2) become

$$
\Lambda=-\frac{n(n-1)}{4 l^{2}} \quad \text { and } \quad \hat{\alpha}_{2}=\frac{l^{2}}{2} .
$$

and so asymptotically Lifshitz solutions exist in the vacuum of Gauss-Bonnet gravity $\left(\alpha_{3}=0\right)$ as well. The cosmological constant here is half that of an AdS spacetime. If we set $\Lambda=0$ then a Lifshitz solution exists in third order Lovelock gravity provided

$$
\hat{\alpha}_{2}=2 l^{2}, \quad \hat{\alpha}_{3}=l^{4} .
$$

Thus, one may have Lifshitz solutions in Lovelock gravity without matter, demonstrating that its higher curvature terms can, for the proper choice of Lovelock coefficients, have the desired effect that matter fields induce.

The preceding analysis was for $k=0$. For $k= \pm 1$ we find for any value of $z$ that

$$
g(r)=1+\frac{k l^{2}}{r^{2}}
$$

furnishes an exact Lifshitz solution to Lovelock gravity without matter, provided one of the above conditions (3.2), (3.3) is satisfied, with the function $f(r)$ undetermined by the field equations (though boundary conditions constrain it to asymptote to 1 ). We can choose $f(r)=g(r)$. This is a naked singularity for $k=1$ but is an asymptotically Lifshitz black hole for $k=-1$. The arbitrariness of $f(r)$ is due to a degeneracy of the field equations; if either of (3.2) or (3.3) hold then the field equations are each proportional to the factor $g(r)-1-k l^{2} / r^{2}$.

This degeneracy of the field equations has been noted previously in 5-dimensional Einstein-Gauss-Bonnet gravity with a cosmological constant [27], where (converting notation appropriately) condition (3.3) was obtained. Here we see that a more general degeneracy occurs in 3rd order Lovelock gravity. We shall see that this degeneracy is lifted when matter is present. We find that no other exact solutions to the field equations exist for these symmetries and asymptotic behavior.

\subsection{Matter Solutions}

Consider next the case of Lifshitz solutions in the presence of a massive gauge field $A^{\mu}$. The metric (3.1) and the gauge field (2.7) with $h(r)=1$ solve the field equation (2.8) provided

$$
m^{2}=\frac{(n-1) z}{l^{2}} .
$$


In order to have asymptotically Lifshitz solutions in 3rd-order Lovelock gravity in the presence of matter, the following constraint

$$
\begin{aligned}
q^{2} & =\frac{2(z-1) L^{4}}{z l^{4}}, \\
\Lambda & =-\frac{\left[(z-1)^{2}+n(z-2)+n^{2}\right] L^{4}+n(n-1)\left(\hat{\alpha}_{2} l^{2}-2 \hat{\alpha}_{3}\right)}{2 l^{6}}
\end{aligned}
$$

must hold for the cosmological constant and charge where we define

$$
L^{4} \equiv l^{4}-2 l^{2} \hat{\alpha}_{2}+3 \hat{\alpha}_{3}
$$

for simplicity and we use this definition throughout the paper. Since $q^{2}>0$ we obtain the constraint

$$
\frac{\hat{\alpha}_{2}}{l^{2}}<\frac{1}{2}+3 \frac{\hat{\alpha}_{3}}{2 l^{4}}
$$

which in turn yields

$$
-\frac{(z-1)(n+z-1)\left(l^{4}+3 \hat{\alpha}_{3}\right)+n(n-1)\left(l^{4}+\hat{\alpha}_{3}\right)}{2 l^{6}} \leq \Lambda<-\frac{n(n-1)}{4 l^{2}}\left(1-\frac{\hat{\alpha}_{3}}{l^{4}}\right)
$$

provided $\hat{\alpha}_{2} \geq 0$. Equation (3.10) shows that for $\hat{\alpha}_{3}<l^{4}$ the cosmological constant is negative. For $\hat{\alpha}_{3}>l^{4}$, the cosmological constant can be positive provided

$$
\frac{(z-1)(n+z-1)\left(l^{4}+3 \hat{\alpha}_{3}\right)+n(n-1)\left(l^{4}+\hat{\alpha}_{3}\right)}{[2(z-1)(z+n-1)+n(n-1)] l^{4}}<\frac{\hat{\alpha}_{2}}{l^{2}}<\frac{1}{2}+3 \frac{\hat{\alpha}_{3}}{2 l^{4}},
$$

where the last inequality comes from the condition (3.9).

Note that for Einstein gravity $\left(\hat{\alpha}_{2}=\hat{\alpha}_{3}=0\right) L=l$ and the above conditions reduce to those which are given in [14] for $n=3$. In Gauss-Bonnet gravity $\left(\alpha_{3}=0\right)$, these conditions become

$$
\begin{aligned}
q^{2} & =\frac{2(z-1)\left(l^{2}-2 \hat{\alpha}_{2}\right)}{z l^{2}}, \\
\Lambda & =-\frac{\left[(z-1)^{2}+n(z-2)+n^{2}\right]\left(l^{2}-2 \hat{\alpha}_{2}\right)+n(n-1) \hat{\alpha}_{2}}{2 l^{4}} .
\end{aligned}
$$

where now $l^{2}$ must be larger than $2 \hat{\alpha}_{2}$. If $\hat{\alpha}_{2}=l^{2} / 2$, then the charge $q$ becomes zero and the conditions (3.11) reduce to the conditions (3.3) as expected. More generally, if $L=0$, then $q$ vanishes and the conditions (3.7) reduce to the conditions (3.2) as expected.

In the absence of a cosmological constant, the Lifshitz solution exists provided

$$
\begin{aligned}
q^{2} & =\frac{2 n(n-1)(z-1)\left(\hat{\alpha}_{2}-2 l^{2}\right)}{z l^{2}\left[3(z-1)^{2}+3 n z+n(n-4)\right]} \\
\hat{\alpha}_{3} & =\frac{\left(2 \hat{\alpha}_{2}-l^{2}\right)\left[(z-1)^{2}+n z\right]+n\left[(n-3) \hat{\alpha}_{2}-(n-2) l^{2}\right]}{\left[3(z-1)^{2}+3 n z+n(n-4)\right]} l^{2} .
\end{aligned}
$$

In this case the conditions (3.12) reduce to the condition (3.4) if $\hat{\alpha}_{2}=2 l^{2}$, leading to $q=0$ and the absence of matter. 


\section{Asymptotic Lifshitz Black holes}

In this section we seek black hole solutions in 3rd-order Lovelock gravity that are asymptotically Lifshitz. We therefore consider the field equations (2.8)-(2.10) with the conditions (3.7), but with the more general ansatz (2.5) and (2.7).

\subsection{Series solutions near the horizon}

We now consider the near-horizon behavior of such solutions. Requiring that $f(r)$ and $g(r)$ go to zero linearly, that is

$$
\begin{aligned}
& f(r)=f_{1}\left\{\left(r-r_{0}\right)+f_{2}\left(r-r_{0}\right)^{2}+f_{3}\left(r-r_{0}\right)^{3}+f_{4}\left(r-r_{0}\right)^{4}+\ldots\right\}, \\
& g(r)=g_{1}\left(r-r_{0}\right)+g_{2}\left(r-r_{0}\right)^{2}+g_{3}\left(r-r_{0}\right)^{3}+g_{4}\left(r-r_{0}\right)^{4}+\ldots, \\
& h(r)=f_{1}^{1 / 2}\left\{h_{0}+h_{1}\left(r-r_{0}\right)+h_{2}\left(r-r_{0}\right)^{2}+h_{3}\left(r-r_{0}\right)^{3}+h_{4}\left(r-r_{0}\right)^{4}+\ldots\right\},
\end{aligned}
$$

and inserting these expansions into the equations of motion arising from Eqs. (2.8)(2.9) with the conditions (3.7), and solving for the various coefficients, we find that $h_{0}=0$. This is consistent with the fact that the flux $d A$ should go to a constant at the horizon. Also, one may note that by scaling time we can adjust the constant $f_{1}$ by an overall multiplicative factor (note the use of $f_{1}^{1 / 2}$ in the expansion of $h(r)$ as well, which is due to $d t$ in the one-form $A$ ). We find the following constraint on the 1st order constants

$$
\begin{aligned}
g_{1}= & \frac{z}{r_{0}^{3}}\left\{3 \hat{\alpha}_{3}\left[h_{1}^{2}(z-1) r_{0}^{5}+k^{2} z(n-1) l^{4}\right]\right. \\
& \left.+2 r_{0}{ }^{2} l^{2} \hat{\alpha}_{2}\left[-h_{1}{ }^{2}(z-1) r_{0}{ }^{3}+l^{2} k z(n-1)\right]+r_{0}{ }^{4} l^{4}\left[h_{1}{ }^{2}(z-1) r_{0}+z(n-1)\right]\right\}^{-1} \\
& \times\left\{\left\{\left[3(z-1)^{2}+3 n z++n(n-4)\right] r_{0}{ }^{6}+k(n-1)(n-6) l^{6}\right\} \hat{\alpha}_{3}\right. \\
& -r_{0}{ }^{2} l^{2} \hat{\alpha}_{2}\left\{\left[2(z-1)^{2}+2 n z+n(n-3)\right] r_{0}{ }^{4}+k^{2} l^{4}(n-1)(n-4)\right\} \\
& \left.+r_{0}{ }^{4} l^{4}\left[(z-1)^{2}+n z+n(n-1)\right] r_{0}{ }^{2}+(n-1)(n-2) k l^{2}\right\},
\end{aligned}
$$

which means that not all boundary conditions are allowed. Note that here the coefficient $h_{1}$ is arbitrary, and should be chosen suitable in order to explore the numerical solutions. The higher order coefficients may be found easily, but their expressions are very lengthy and so we don't write them here for reasons of economy.

One may also investigate the behavior of the metric functions at large $r$. We relegate this to the Appendix, where we show that the powers of $1 / r$ for $k=0$ are in general non-integer.

\subsection{Numeric Solutions}

In order to find the numeric black hole solutions of the system given by Eqs. (2.8)(2.10) with the conditions (3.7), we define

$$
\frac{d h}{d r} \equiv j(r),
$$


and find the first derivatives of $j(r), f(r)$ and $g(r)$ as

$$
\begin{aligned}
& \frac{d j}{d r}=\frac{(n-1) z h-[z(n+z-2) h+(n+2 z-1) r j] g}{r^{2} g}+\frac{(z-1) L^{4}(z h+r j) r^{4} h^{2}}{r^{2} f g H}, \\
& \frac{d f}{d r}=\frac{1}{(n-1) z r^{3} g H}\left\{-(n-1)[n+6(z-1)] z \hat{\alpha}_{3} r^{6} f g^{3}\right. \\
& +(n-1) z l^{2} r^{4}\left[3 k \hat{\alpha}_{3}(n+4 z-6)+(n+4 z-4) \hat{\alpha}_{2} r^{2}\right] f g^{2} \\
& -(n-1) z l^{4} r^{2}\left[3(n+2 z-6) k^{2} \hat{\alpha}_{3}+2(n+2 z-4) k \hat{\alpha}_{2} r^{2}+(n+2 z-2) r^{4}\right] f g \\
& +z \hat{\alpha}_{3}\left\{\left[3(z-1)^{2}+3 n z+n(n-4)\right] r^{6}+(n-1)(n-6) k l^{6}\right\} f \\
& -z \hat{\alpha}_{2} l^{2} r^{2}\left\{\left[2(z-1)^{2}+2 n z+n(n-3)\right] r^{4}-(n-1)(n-4) k^{2} l^{4}\right\} f \\
& +z l^{4} r^{4}\left\{\left[(z-1)^{2}+n z+n(n-1)\right] r^{2}+(n-1)(n-2) k l^{2}\right\} f \\
& \left.-(z-1) L^{4} r^{6}\left[(z h+r j)^{2} g+(n-1) z h^{2}\right]\right\} \text {, } \\
& \frac{d g}{d r}=\frac{1}{(n-1) z r^{3} f H}\left\{-n(n-1) z \hat{\alpha}_{3} r^{6} f g^{3}+(n-1) z l^{2} r^{4}\left[3 k \hat{\alpha}_{3}(n-2)+n \hat{\alpha}_{2} r^{2}\right] f g^{2}\right. \\
& -(n-1) z l^{4} r^{2}\left[3(n-4) k^{2} \hat{\alpha}_{3}+2(n-2) k \hat{\alpha}_{2} r^{2}+n r^{4}\right] f g \\
& +z \hat{\alpha}_{3}\left\{\left[3(z-1)^{2}+3 n z+n(n-4)\right] r^{6}+(n-1)(n-6) k l^{6}\right\} f \\
& -z \hat{\alpha}_{2} l^{2} r^{2}\left\{\left[2(z-1)^{2}+2 n z+n(n-3)\right] r^{4}-(n-1)(n-4) k^{2} l^{4}\right\} f \\
& +z l^{2} r^{2}\left\{\left[(z-1)^{2}+n z+n(n-1)\right] r^{2}+(n-1)(n-2) k l^{2}\right\} f \\
& \left.-(z-1) L^{4} r^{6}\left[(z h+r j)^{2} g+(n-1) z h^{2}\right]\right\} \text {, }
\end{aligned}
$$

where $H$ is

$$
H=3 \hat{\alpha}_{3}\left(k l^{2}-r^{2} g\right)^{2}+\hat{\alpha}_{2} l^{2} r^{2}\left(k l^{2}-r^{2} g\right)+l^{4} r^{4} .
$$

Now having a system of 4 first order ordinary differential equations, one may explore the numeric solutions by choosing suitable initial conditions for $f\left(r_{0}\right), g\left(r_{0}\right), h\left(r_{0}\right)$ and $j\left(r_{0}\right)$, where $r_{0}$ is the radius of horizon. This can be done by using the method explained in [13]. First, we apply the numerical method for $z=1$ which can be solved exactly, and compare the numerical solution to the exact one. Indeed, for $z=1$, the charge vanishes, and the problem reduces to the case of black holes in third order Lovelock gravity with specific choices of Lovelock coefficients. In this case, we obtain the exact solutions of Lovelock gravity [28], which have $f(r)=g(r)$. The diagrams of the functions $f(r)$ and $g(r)$ versus $r$ for $z=1$ with $k=0$ and $k= \pm 1$ have been shown in Fig 4.1. In this figure the exact solutions have also been shown. As one can see from the plots, the numerically obtained functions $f(r)$ and $g(r)$ are equal and both of them are exactly lie on the exact solution within limits of numerical precision. We regard this as a good test of our methods. Note that for $k=0,-1$ and $z=1$ (the solution without matter), the metric functions increase from zero at 


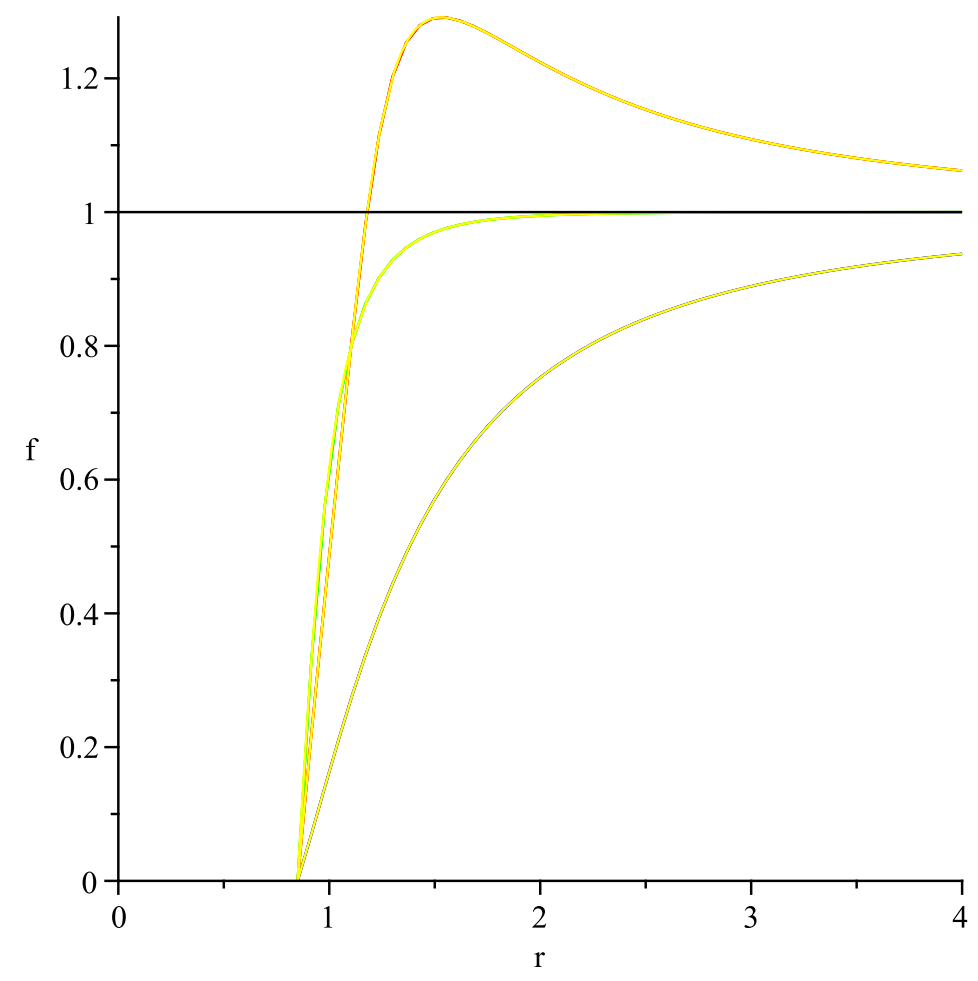

Figure 4.1: $f(r), g(r)$ and exact solution versus $r$ for $n=6, l=1, z=1, \hat{\alpha}_{2}=.25$, $\hat{\alpha}_{3}=.3$ and $r_{0}=0.85$ for $k=1$ (top), $k=0$ (middle) and $k=-1$ (bottom). Note that $f(r), g(r)$ and the exact solution lie on each other for each $k$.

$r=r_{0}$ to 1 at $r=\infty$. However for $k=1$ and $z=1$, the metric functions grow to be larger than unity at intermediate values of $r$ and asymptote to 1 at infinity. Now, we consider the solutions for $z \neq 1$, for which matter exists, where we must solve the system of equations numerically. Figure 4.2 shows the function $f(r)$ as a function of $r$ for a small black hole of Einstein, Gauss-Bonnet and Lovelock gravity with $z=1$ and $z=2$. As one can see from this figure, while $f(r)$ for Einstein, Gauss-Bonnet and Lovelock with $z=1$ (solution without matter) are almost the same, they are different for $z=2$ for the same values of $\alpha_{2}$ and $\alpha_{3}$. Furthermore, for $z=2$, the metric function $f(r)$ rapidly increases to values larger than 1, eventually asymptoting to 1 as $r \rightarrow \infty$, while for $z=1$ this function monotonically increases from zero at $r=r_{0}$ to 1 at $r=\infty$. Thus, it appears that asymptotic Lifshitz solutions are more sensitive than are their $z=1$ (AdS) counterparts to the corrections induced by Lovelock gravity. This feature also occurs for large black holes with $k=-1$ (see Fig. 4.3). Note that for $k=-1$, the function $f(r)$ in Gauss-Bonnet gravity is larger than that in third order Lovelock gravity. This is due to the fact that the slope of the function $f(r)$ decreases as $\hat{\alpha}_{3}$ increases (see the terms in $d f / d r$ with the factor $\hat{\alpha}_{3}$ in Eq. (4.5) with $k=-1$, which are negative). Figures 4.4 4.5 show that this fact also occurs for the small and large black holes with $k=0$. For the case of $k=1$, one 


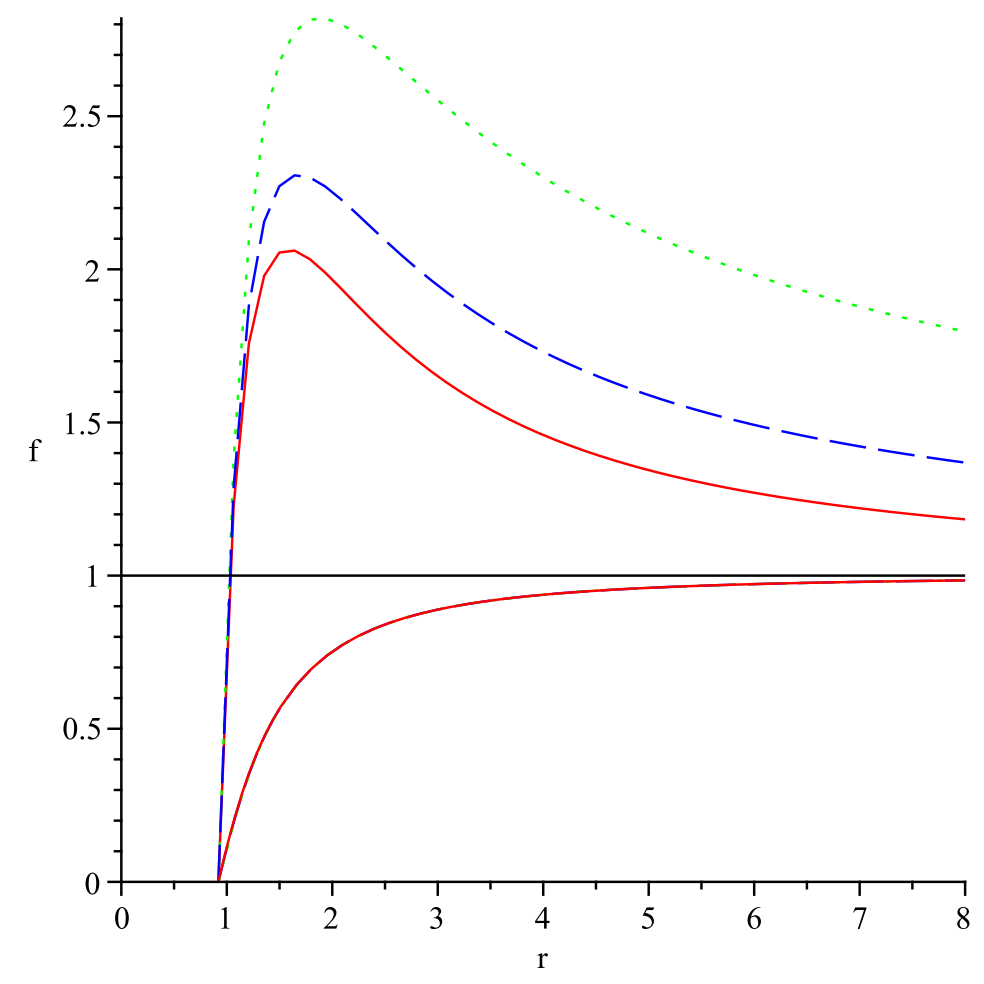

Figure 4.2: $f(r)$ versus $r$ for $k=-1, n=6, l=1, \hat{\alpha}_{2}=.25, \hat{\alpha}_{3}=.3, r_{0}=0.92$ in 3rd order Lovelock, Gauss-Bonnet and Einstein gravities red (solid), green (dotted) and blue (dashed), respectively. The three upper curves are $f(r)$ for $z=2$ and the three lower curves, which are nearly identical and so lie on top of each other, correspond to $z=1$.

can see from Figs 4.6 and 4.7 that both the asymptotic AdS and Lifshitz black holes are different for various order of Lovelock gravities, although the difference between the metric function $f(r)$ for various order of Lovelock gravity becomes more relevant as $z$ increases.

We find that the dependence on $z$ of $g(r)$ is not as significant as for $f(r)$. For this and reasons of economy, we plot only the function $g(r)$ in one case (see Fig. 4.8). We also find that $g(r)$ is monotonically increasing for topological black holes with $k=0$, whereas (as noted previously) $f(r)$ has a maximum larger than 1 at $r$ several times $r_{0}$.

We pause to comment on the relative importance of the various terms in the Lovelock action. While there is no essential problem in considering all terms to be similar in magnitude, we obtain some insight into Lovelock gravity by treating it as an approximation (in powers of the curvature) to a full quantum theory of gravity. For simplicity we shall consider solutions obtained in this paper for $k=0$, which asymptote to the Lifshitz background (3.1). In this case the $p$ th order Lagrangian of 


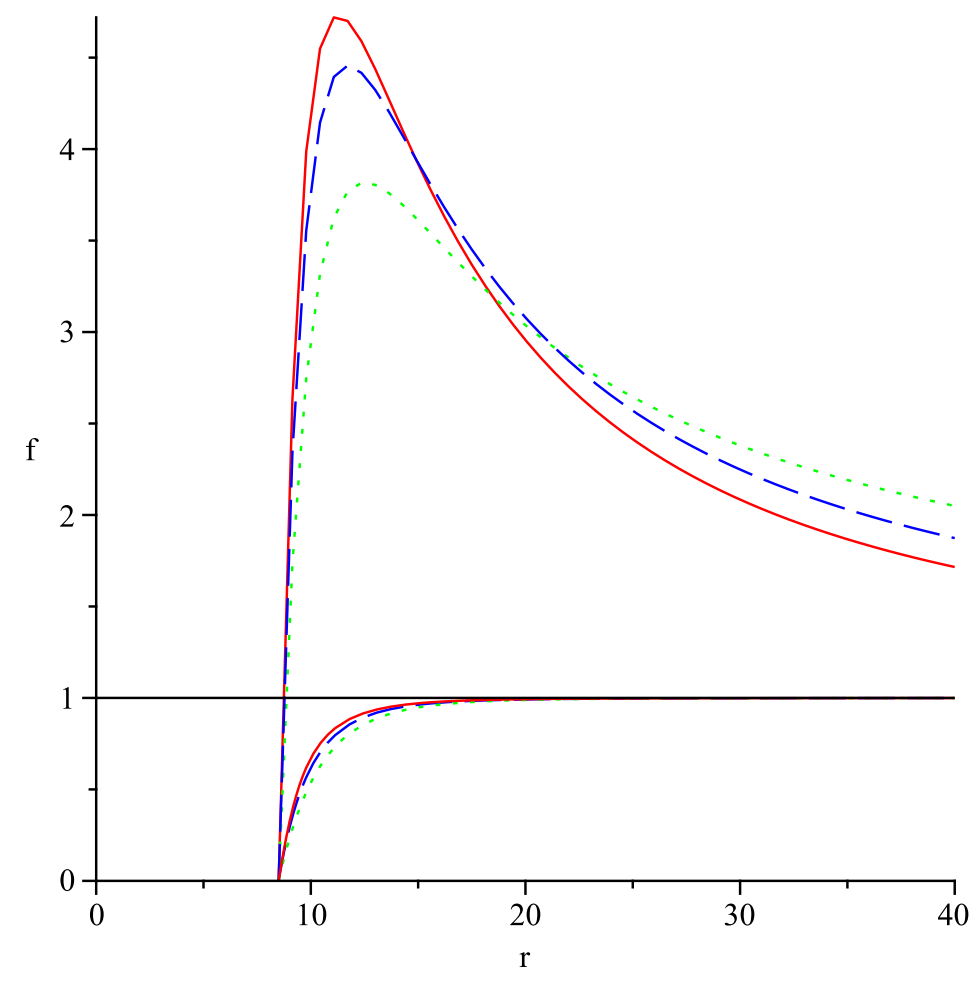

Figure 4.3: $f(r)$ versus $r$ for $k=-1, n=6, l=1, \hat{\alpha}_{2}=.25, \hat{\alpha}_{3}=.3, r_{0}=8.5$ in 3rd order Lovelock, Gauss-Bonnet and Einstein gravities red (solid), green (dotted) and blue (dashed), respectively. The three upper curves are $f(r)$ for $z=2$ and the three lower curves correspond to $z=1$.

Lovelock gravity for the metric (2.5) may be written as:

$$
\mathcal{L}_{p}^{\prime}=(-1)^{p-1} \frac{(n-1)}{(2 p-1) l^{z+2 p-1} r^{(2 p-1)(z-1)}}\left(\frac{g}{f}\right)^{p-1 / 2} \frac{d}{d r}\left\{r^{n+2 p(z-1)} f^{p}\right\}
$$

where $\mathcal{L}_{p}^{\prime}=\left(\alpha_{p} / \hat{\alpha}_{p}\right) \sqrt{-g} \mathcal{L}_{p}$. The above expression for the Lagrangian is correct for any value of $p$, though in this paper we have considered only $p \leq 3$. The Lagrangian (4.6) can be evaluated on the solutions at large $r$ by the use of the expansions of the metric functions which are given in the Appendix. It is a matter of calculation to show that the ratio $\mathcal{L}_{p+1}^{\prime} / \mathcal{L}_{p}^{\prime}$ at large $r$ is

$$
\frac{\mathcal{L}_{p+1}^{\prime}}{\mathcal{L}_{p}^{\prime}}=-\frac{(2 p-1)[n+2(p+1)(z-1)]}{(2 p+1)[n+2 p(z-1)] l^{2}}
$$

and so $\mathcal{L}_{p+1}^{\prime}<\mathcal{L}_{p}^{\prime} / l^{2}$. 


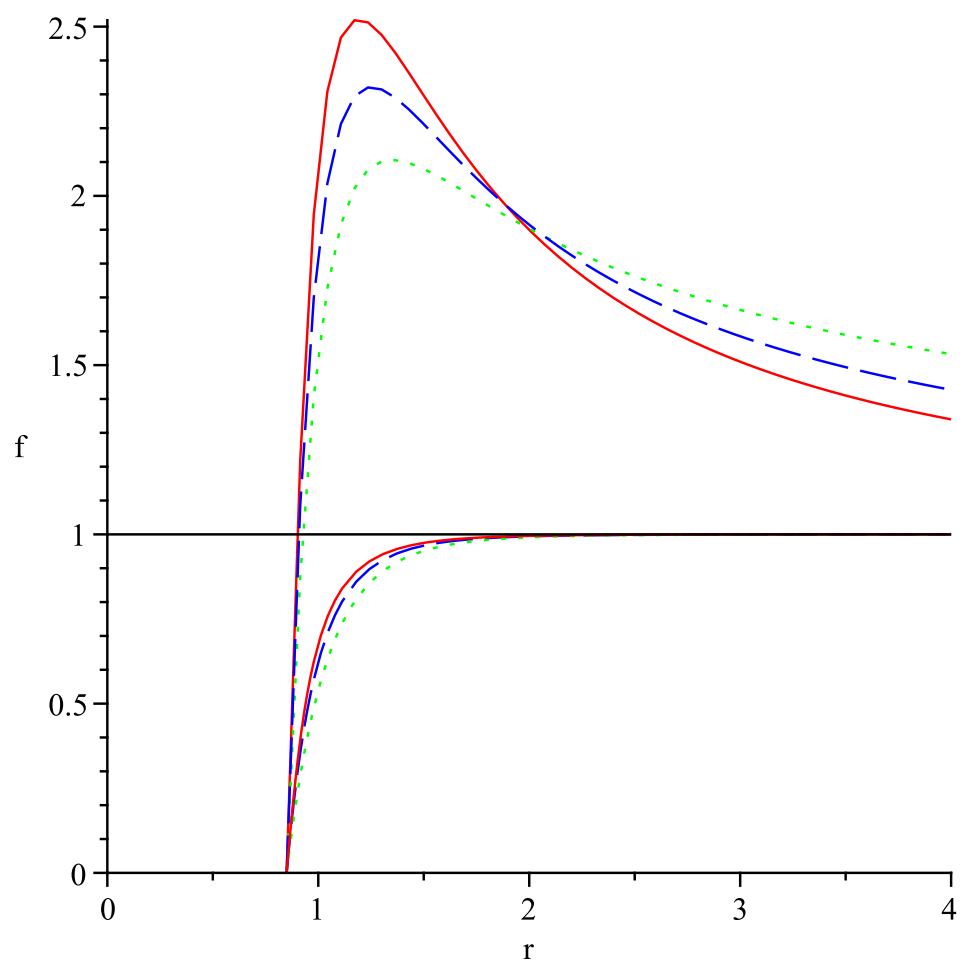

Figure 4.4: $f(r)$ versus $r$ for $k=0, n=6, l=1, \hat{\alpha}_{2}=.25, \hat{\alpha}_{3}=.3, r_{0}=0.85$ in $3 r d$ order Lovelock, Gauss-Bonnet and Einstein gravities red (solid), green (dotted) and blue (dashed), respectively. The three upper curves are $f(r)$ for $z=2$ and the three lower curves correspond to $z=1$.

\section{Thermodynamics of black holes}

The entropy of a black hole in Lovelock gravity is 29]

$$
S=\frac{1}{4} \sum_{k=1}^{p} k \alpha_{k} \int d^{n-1} x \sqrt{\tilde{g}} \tilde{\mathcal{L}}_{k-1}
$$

where the integration is done on the $(n-1)$-dimensional spacelike hypersurface of the Killing horizon with induced metric $\tilde{g}_{\mu \nu}$ (whose determinant is $\tilde{g}$ ), and $\tilde{\mathcal{L}}_{k}$ is the $k$ th order Lovelock Lagrangian of $\tilde{g}_{\mu \nu}$. It is a matter of calculation to show that the entropy of a black hole per unit volume of the horizon in third order Lovelock gravity is

$$
S=\frac{r_{0}^{n-1}}{4}\left(1+\frac{2 k(n-1) \hat{\alpha}_{2}}{(n-3) r_{0}^{2}}+\frac{3 k^{2}(n-1) \hat{\alpha}_{3}}{(n-5) r_{0}^{4}}\right) .
$$

This reduces to the area law of entropy for $\hat{\alpha}_{2}=\hat{\alpha}_{3}=0$. 


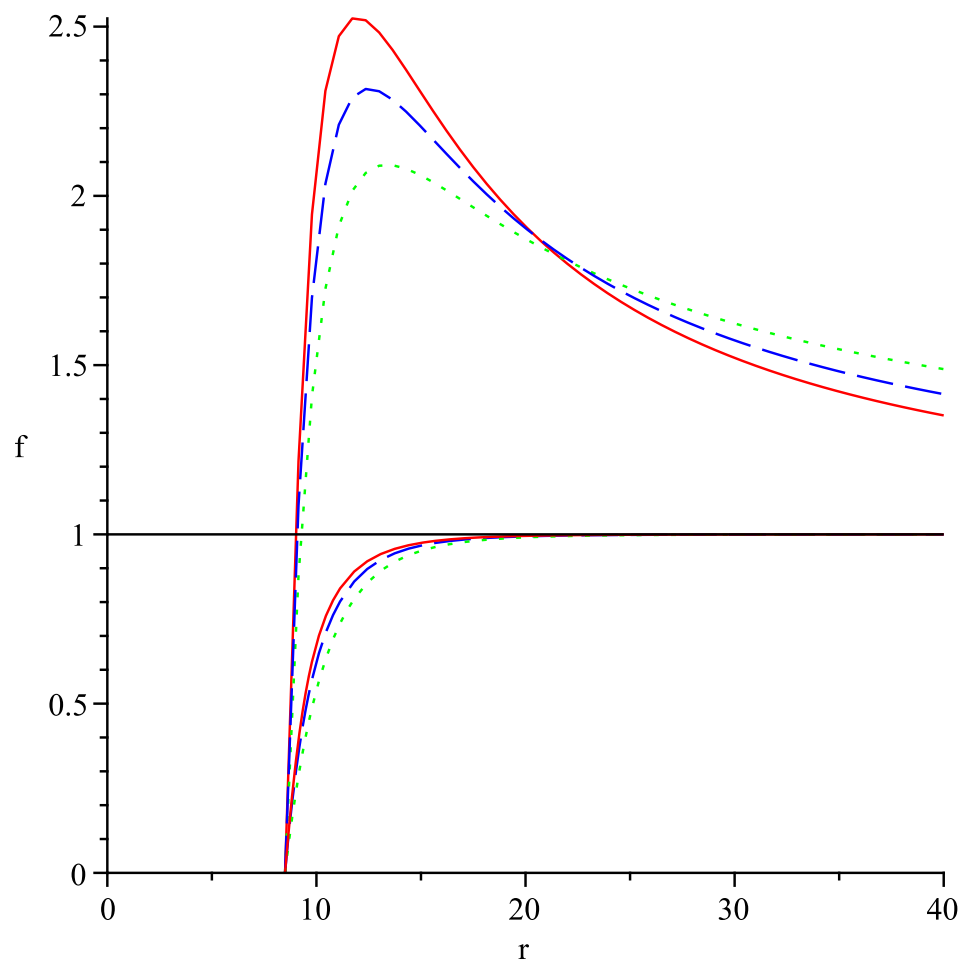

Figure 4.5: $f(r)$ versus $r$ for $k=0, n=6, l=1, \hat{\alpha}_{2}=.25, \hat{\alpha}_{3}=.3, r_{0}=8.5$ in $3 \mathrm{rd}$ order Lovelock, Gauss-Bonnet and Einstein gravities red (solid), green (dotted) and blue (dashed), respectively. The three upper curves are $f(r)$ for $z=2$ and the three lower curves correspond to $z=1$.

One can obtain the temperature of the event horizon by using standard Wickrotation methods, yielding the result

$$
T=\left(\frac{r^{z+1} \sqrt{f^{\prime} g^{\prime}}}{4 \pi l^{z+1}}\right)_{r=r_{0}} .
$$

For the case of asymptotic Lifshitz black hole with (identified) hyperbolic horizon, the temperature of the exact solution (3.5) with horizon radius $r_{0}=l$ in vacuum is

$$
T=\frac{1}{2 \pi l} \text {. }
$$

The temperature of more general Lovelock-Lifshitz black holes can be calculated numerically. First, we review the thermodynamics of asymptotic AdS black holes $(z=1)$ to compare it with the asymptotic Lifshitz black holes. As one can see from figure 5.1, the logarithm of the temperature of black holes versus the logarithm of the entropy for large black holes is linear, while for small black holes this occurs only in the case of $k=0$. For $k=-1$, one encounters with an extreme black hole in Einstein (blue dashed line) and Lovelock gravity (blue solid line). However, the 


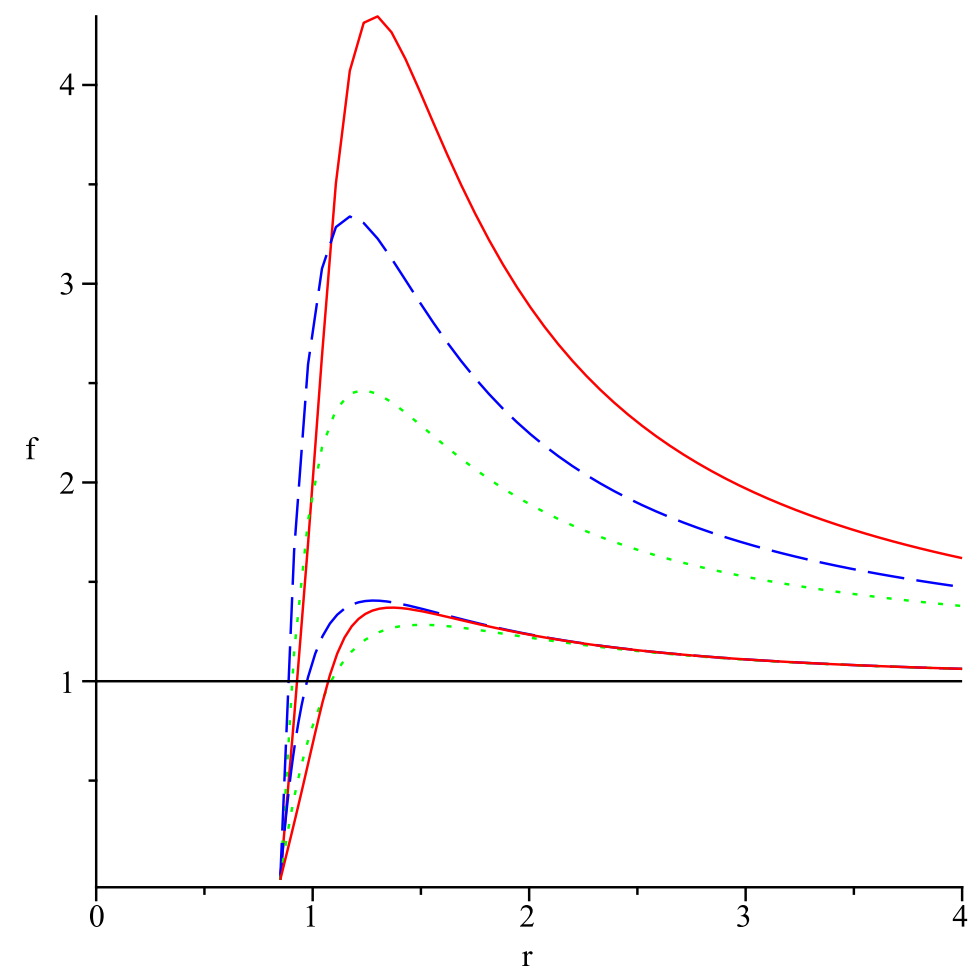

Figure 4.6: $f(r)$ versus $r$ for $k=1, n=6, l=1, \hat{\alpha}_{2}=.25, \hat{\alpha}_{3}=.3, r_{0}=0.85$ in 3 rd order Lovelock, Gauss-Bonnet and Einstein gravities red (solid), green (dotted) and blue (dashed), respectively. The three upper curves are $f(r)$ for $z=2$ and the three lower curves correspond to $z=1$.

horizon radius of the extreme black hole of Lovelock gravity is smaller than that of Einstein gravity. Note that the slopes of the graphs of $\log T$ versus $\log S$ for small black holes of Einstein and Lovelock gravity with $k=1$ are negative, and therefore they are unstable. The unstable phase in Lovelock gravity occurs for a smaller radius black hole than in Einstein theory. Plotting $\log T$ versus $\log S$ for the case of asymptotic Lifshitz black holes with $z=2$, we see from Fig. 5.2 that the temperature of black holes in Lovelock gravity with given entropy $S$ is smaller than the temperature of black holes of Einstein gravity with the same entropy. Conversely, at a given temperature the entropy of the Lovelock black holes - extremal and nonextremal - is larger than for the Einstein ones for both $z=1$ and $z=2$. The horizon radii of extreme black holes in both Einstein and Lovelock gravity for $k=-1$ with $z=2$ are smaller than their $z=1$ counterparts, and for a given $z$ smaller in Lovelock gravity than in Einstein gravity.

Numerical calculations show that there is no unstable phase for $z=2$ black holes for $k=1$. We also find that for $k=0$ and the large black holes for $k= \pm 1$, the 


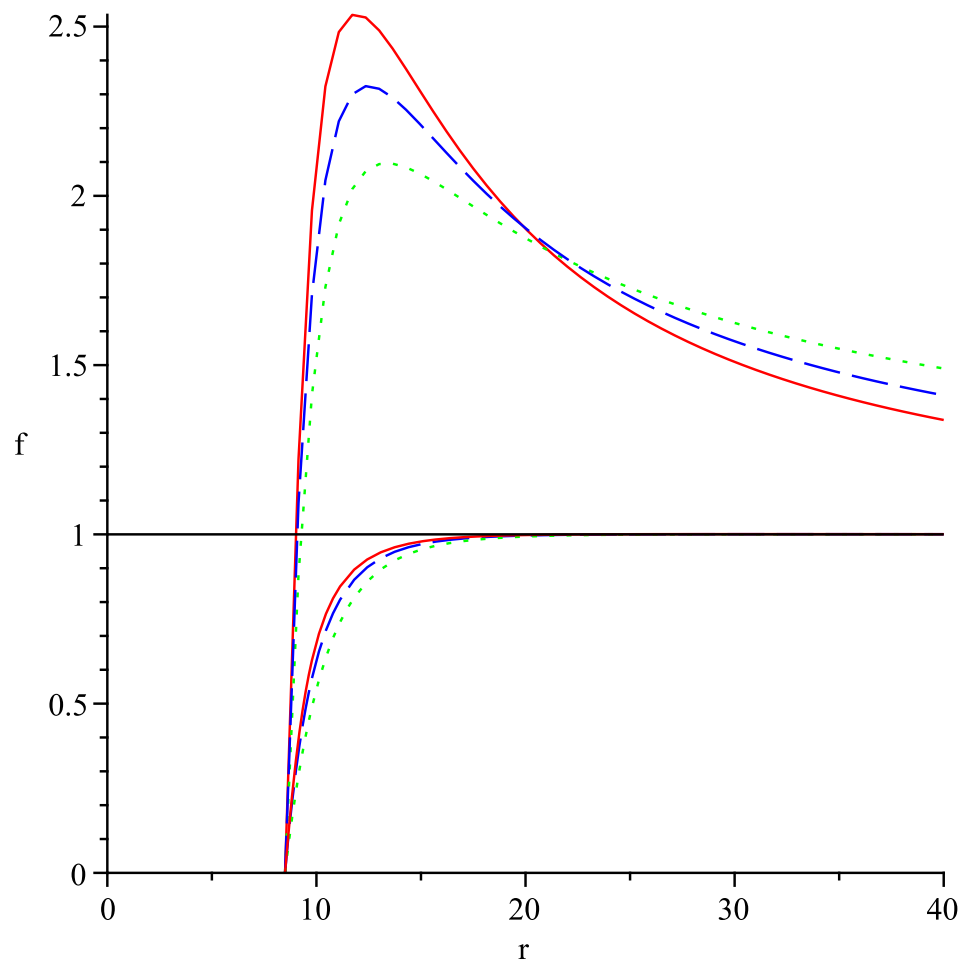

Figure 4.7: $f(r)$ versus $r$ for $k=1, n=6, l=1, \hat{\alpha}_{2}=.25, \hat{\alpha}_{3}=.3, r_{0}=8.5$ in 3 rd order Lovelock, Gauss-Bonnet and Einstein gravities red (solid), green (dotted) and blue (dashed), respectively. The three upper curves are $f(r)$ for $z=2$ and the three lower curves correspond to $z=1$.

temperature is proportional to $r_{0}^{z}$, while the entropy is proportional to $r_{0}^{n-1}$, yielding

$$
T \propto S^{z /(n-1)} .
$$

for the dependence of $T$ on $S$. In order to say more about the temperature, one should compute the energy density of these black holes using (for example) the counterterm method. While some work has been done on this for asymptotic Lifshitz solutions of Einstein gravity [30, 31], the corresponding formalism needs to be developed for Lovelock gravity, an endeavour that we hope to address in the future.

\section{Rotating Lovelock-Lifshitz solutions}

In this section we endow the Lifshitz spacetime with a global rotation. We first consider the Lifshitz solution with one rotation parameter. We write the metric as

$$
d s^{2}=-\frac{r^{2 z}}{l^{2 z}} d t^{2}+\frac{l^{2} d r^{2}}{r^{2}}+r^{2} d \theta_{1}^{2}+r^{2} \sum_{i=2}^{n-1} d \theta_{i}^{2}
$$




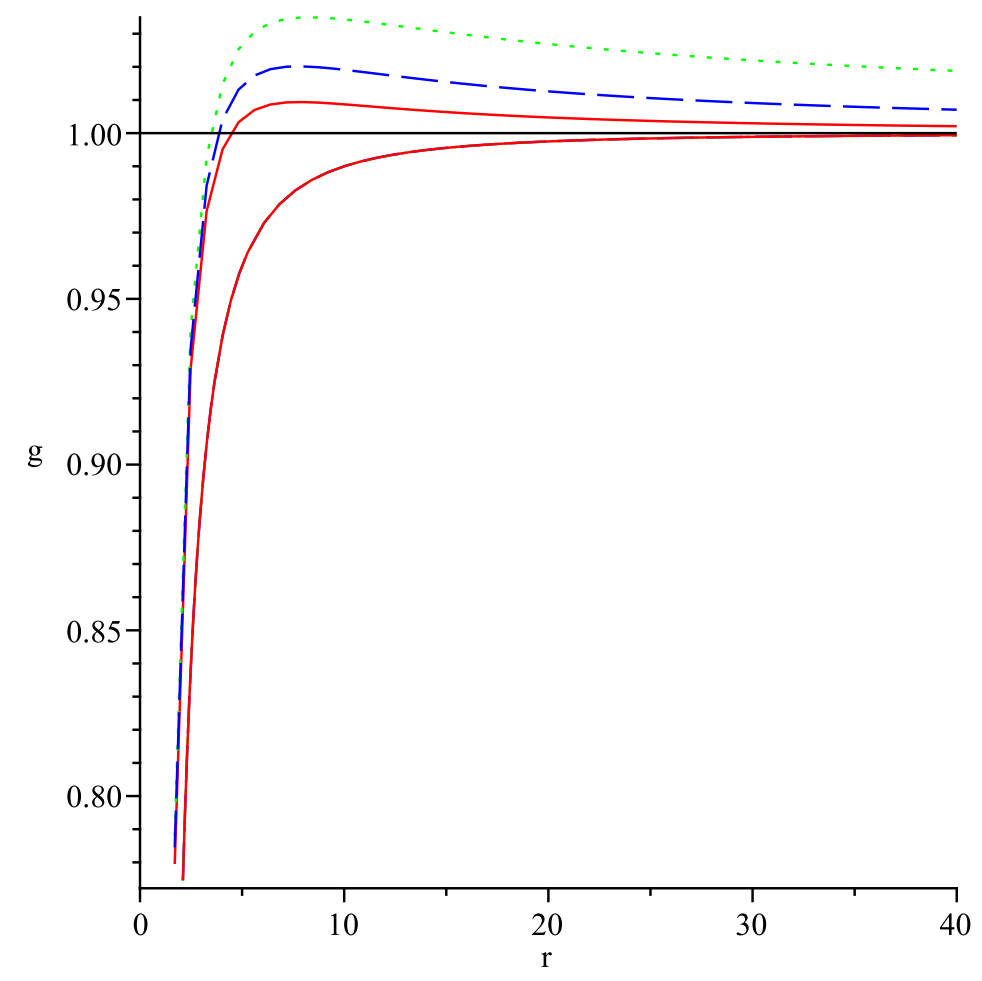

Figure 4.8: $g(r)$ versus $r$ for $k=-1, n=6, l=1, \hat{\alpha}_{2}=.25, \hat{\alpha}_{3}=.3, r_{0}=0.85$ in 3rd order Lovelock, Gauss-Bonnet and Einstein gravities red (solid), green (dotted) and blue (dashed), respectively. The three upper curves are $f(r)$ for $z=2$ and the three lower curves, which lie on each other, correspond to $z=1$.

In order to add angular momentum to the spacetime, we perform the following rotation boost in the $t-\theta_{1}$ plane:

$$
t \mapsto \Xi t-a \theta_{1} \quad \theta_{1} \mapsto \Xi \theta_{1}-\frac{a}{l^{2}} t
$$

where $a$ is the rotation parameter and $\Xi=1+a^{2} / l^{2}$. Substituting eq. (6.2) into eq. (6.1) we obtain

$$
d s^{2}=-\frac{r^{z}}{l^{z}}\left(\Xi d t-a d \theta_{1}\right)^{2}+\frac{l^{2} d r^{2}}{r^{2}}+r^{2}\left(\frac{a}{l^{2}} d t-\Xi d \theta_{1}\right)^{2}+r^{2} \sum_{i=2}^{n-1} d \theta_{i}^{2} .
$$

The transformation (6.2) generates a new metric if $\theta_{1}$ is periodically identified since the transformation (6.2) can be done locally but not globally 32]. The periodic nature of $\theta_{1}$ allows the metrics (6.1) and (6.3) to be locally mapped into each other but not globally, and so they are distinct. It is a matter of straightforward calculation to show that the metric (6.3) is a solution of Lovelock gravity, provided one of the conditions (3.2)-(3.3) holds. Furthermore the metric (6.3) is also a solution to the 


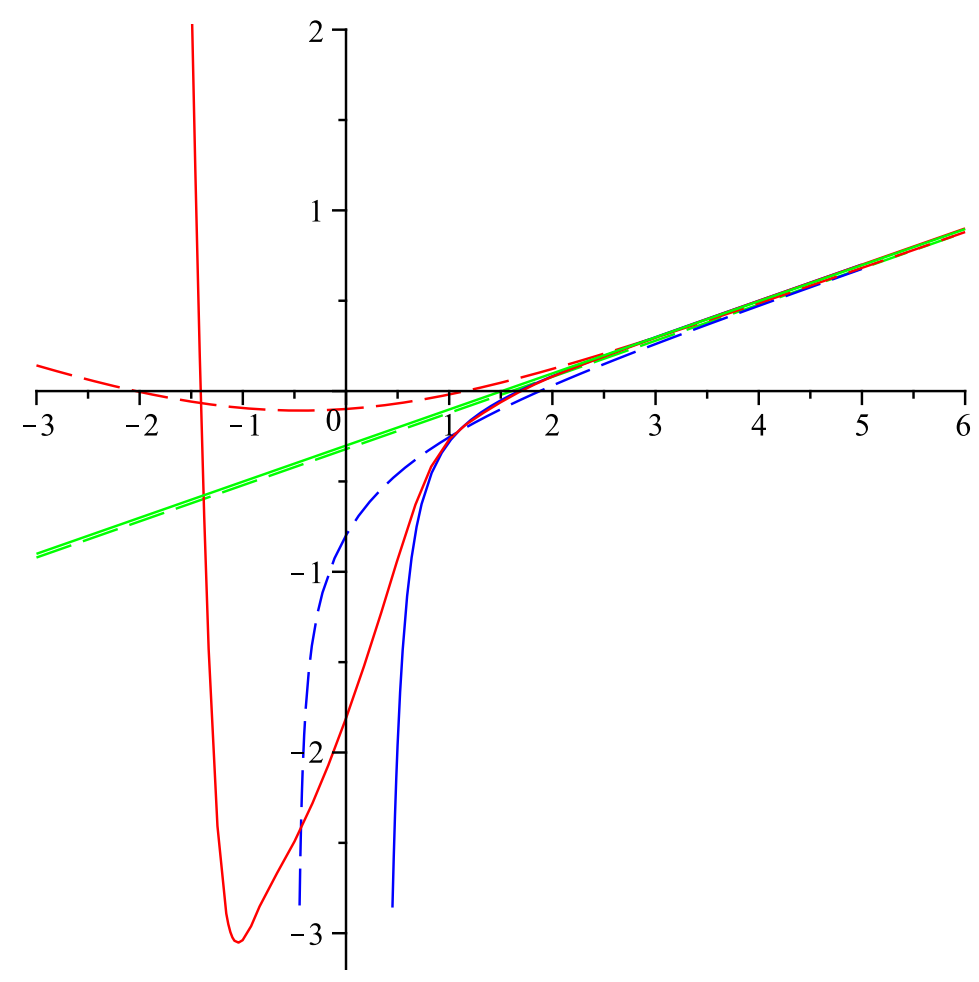

Figure 5.1: $\log T$ versus $\log S$ for $n=6, l=1, z=1$ in Einstein and Lovelock $\left(\hat{\alpha}_{2}=.25\right.$, $\hat{\alpha}_{3}=.3$ ) gravities with $k=1$ (red), $k=0$ (green) and $k=-1$ (blue), where the dashed and solid lines are curves in Einstein and Lovelock gravities, respectively.

field equations in the presence of the vector field

$$
A=q \frac{r^{z}}{l^{z}}\left(\Xi d t-a d \theta_{1}\right)
$$

provided the condition (3.6) and one of the conditions (3.11)-(3.12) are satisfied.

It is straightforward to generalize the metric (6.3) to a metric with more rotation parameters. The rotation group in $(n+1)$ dimensions is $S O(n)$, and therefore the number of independent rotation parameters is $[n / 2]$. The generalized solution with $m \leq[n / 2]$ rotation parameters can be written as

$$
\begin{aligned}
d s^{2}= & -\frac{r^{z}}{l^{z}}\left(\Xi d t-\sum_{i=1}^{m} a_{i} d \theta_{i}\right)^{2}+\frac{r^{2}}{l^{4}} \sum_{i=1}^{m}\left(a_{i} d t-\Xi l^{2} d \theta_{i}\right)^{2} \\
& +\frac{l^{2} d r^{2}}{r^{2}}-\frac{r^{2}}{l^{2}} \sum_{i<j}^{m}\left(a_{i} d \theta_{j}-a_{j} d \theta_{i}\right)^{2}+r^{2} \sum_{i=m+1}^{n-1} d \theta_{i}^{2}
\end{aligned}
$$

where $\Xi=\sqrt{1+\sum_{i}^{m} a_{i}^{2} / l^{2}}$ and the angular coordinates are in the range $0 \leq \theta_{i}<2 \pi$. This generalization can be extended to the Lovelock-Lifshitz $k=0$ black hole since the transformation (6.2) does not change the $r$-dependence of the metric func- 


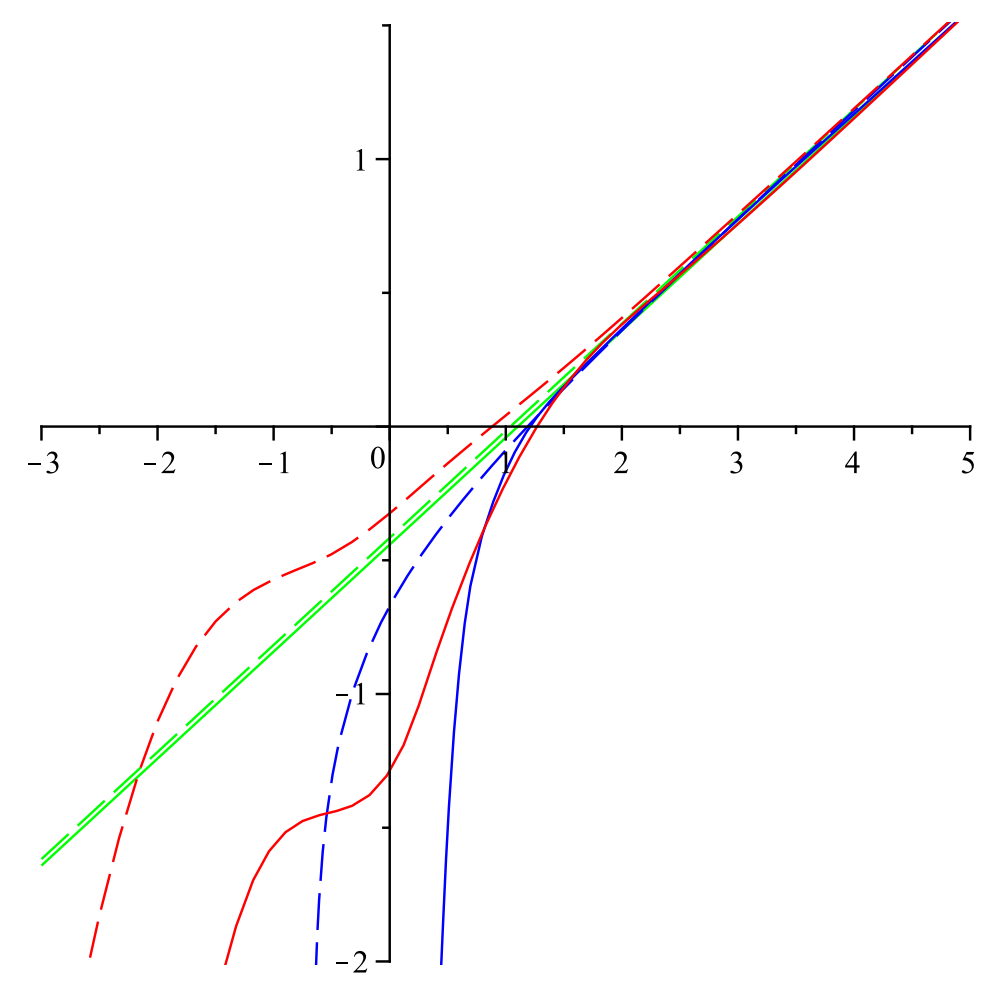

Figure 5.2: $\log T$ versus $\log S$ for $n=6, l=1, z=2$ in Einstein and Lovelock $\left(\hat{\alpha}_{2}=.25\right.$, $\hat{\alpha}_{3}=.3$ ) gravities with $k=1$ (red), $k=0$ (green) and $k=-1$ (blue), where the dashed and solid lines are curves in Einstein and Lovelock gravities, respectively.

tions and the vector field. Hence

$$
\begin{aligned}
d s^{2}= & -\frac{r^{z}}{l^{z}} f(r)\left(\Xi d t-\sum_{i=1}^{k} a_{i} d \theta_{i}\right)^{2}+\frac{r^{2}}{l^{4}} \sum_{i=1}^{k}\left(a_{i} d t-\Xi l^{2} d \theta_{i}\right)^{2} \\
& +\frac{l^{2} d r^{2}}{r^{2} g(r)}-\frac{r^{2}}{l^{2}} \sum_{i<j}^{k}\left(a_{i} d \theta_{j}-a_{j} d \theta_{i}\right)^{2}+r^{2} \sum_{i=k+1}^{n-1} d \theta_{i}^{2},
\end{aligned}
$$

with the vector field

$$
A=q \frac{r^{z}}{l^{z}} h(r)\left(\Xi d t-\sum_{i=1}^{k} a_{i} d \theta_{i}\right),
$$

is a rotating black hole solution to the field equations provided $f(r), g(r)$ and $h(r)$ are chosen to be the functions calculated in section 4.2 for $k=0$.

\section{Concluding Remarks}

It is known that Einstein gravity in the presence of a massive vector field can support Lifshitz solutions [11]. We have demonstrated here that such solutions exist in pure 
Lovelock gravity provided the coupling parameters are chosen appropriately. The higher curvature terms appear to play the role of some kind of matter field, whose nature depends on the constants of the theory. We also found for any value of $z$ an exact vacuum asymptotically Lifshitz solution (3.5) for $k= \pm 1$, which is a black hole for $k=-1$, and a naked singularity for $k=1$. This solution exists in both Gauss-Bonnet and 3rd order Lovelock gravity, depending on the choice of coupling.

We also obtained a broad class of solutions for Lovelock gravity coupled to a massive vector field. After demonstrating that Lovelock gravity can support a Lifshitz solution in the presence of a massive vector field, we searched for asymptotic Lifshitz black holes in the presence of a massive vector field. Our numerically obtained solutions generalize those obtained in Einsteinian gravity coupled to a massive vector field [13, 14, 16]. We found that asymptotic Lifshitz solutions $(z>1)$ are more sensitive to the corrections induced by Lovelock gravity than are their $z=1$ counterparts.

We also considered the thermodynamics of the black hole solutions. We found that, as in the case of asymptotically AdS black holes of Lovelock gravity, one can have an extreme black hole only for $k=-1$. However the horizon radius of both the extreme black holes of Einstein and Lovelock gravity for $k=-1$ with $z=2$ are smaller than their $z=1$ counterparts. That is, the radius of the extreme black holes decreases as $z$ increases both in Einstein and Lovelock gravity. Also, Lovelock terms decrease the radius of extremal black holes compared to their Einsteinian counterparts. The temperature of a Lovelock-Lifshitz black hole with entropy $S$ is smaller than the temperature of a Lifshitz black hole in Einstein gravity with the same entropy. We also found, numerically, that as $z$ increases the temperature of a black hole with a fixed horizon radius increases. Indeed, the temperature is proportional to $r_{0}^{z}$ for black holes with zero curvature horizon and also for large black holes with nonzero horizon curvature. In these two cases the temperature is proportional to $S^{z /(n-1)}$.

We investigated only the cases with $z \geq 2$ and found that these solutions are thermodynamically stable. This fact can be seen by diagrams of temperature versus the entropy. This feature of asymptotically Lifshitz black holes is different from the asymptotically AdS solutions that can have an unstable phase.

Our results demonstrate that higher-order curvature corrections to "Lifshitz holography" are under control and have a sensible physical interpretation. However there is much remaining to explore for these kinds of black holes, including the corrections they induce in the dual boundary theory and a more detailed study of the behavior for larger $n$ and larger $z$. The rotating $k=0$ solutions should have counterparts for the $k= \pm 1$ cases. Work on these areas is in progress.

\section{Appendix}

Here, we investigate the behavior of the metric functions at large $r$. For the case of 
$k=0$ the powers of $1 / r$ may be non-integer, and therefore we consider it separately.

For this case, we use straightforward perturbation theory, writing

$$
\begin{aligned}
& f(r)=1+\varepsilon f_{1}(r), \\
& g(r)=1+\varepsilon g_{1}(r), \\
& h(r)=1+\varepsilon h_{1}(r),
\end{aligned}
$$

and then finding the field equations up to the first order in $\varepsilon$. We obtain

$$
\begin{aligned}
0= & 2 r^{2} h_{1}^{\prime \prime}+2(n+z) r h_{1}^{\prime}+z r\left(g_{1}^{\prime}-f_{1}^{\prime}\right)+2(n-1) z g_{1}, \\
0= & 2(z-1) r h_{1}^{\prime}+(n-1) r g_{1}^{\prime}+[z(z-1)+n(n-1)] g_{1}-(z-1)(n+z-1)\left(f_{1}-2 h_{1}\right), \\
0= & 2(z-1) r h_{1}^{\prime}+(n-1) r g_{1}^{\prime}+[z(z-1)+n(n-1)+2(n-1)(z-1) \mathcal{B}] g_{1} \\
& -(z-1)(z-n+1)\left(f_{1}-2 h_{1}\right)
\end{aligned}
$$

where $\mathcal{B}=\left(l^{4}-4 \hat{\alpha}_{2} l^{2}+9 \hat{\alpha}_{3}\right) / L^{4}$. Note that all the parameters of Lovelock gravity are in $\mathcal{B}$, with $\mathcal{B}=1$ in Einstein gravity. The solution of the above equations may be written as

$$
\begin{aligned}
& h_{1}(r)=C_{1} r^{-(n+z-1)}+r^{-(n+z-1) / 2}\left(C_{2} r^{-\gamma / 2}+C_{3} r^{\gamma / 2}\right), \\
& f_{1}(r)=C_{1} F_{1} r^{-(n+z-1)}+r^{-(n+z-1) / 2}\left(C_{2} F_{2} r^{-\gamma / 2}+C_{3} F_{3} r^{\gamma / 2}\right), \\
& g_{1}(r)=C_{1} G_{1} r^{-(n+z-1)}+r^{-(n+z-1) / 2}\left(C_{2} G_{2} r^{-\gamma / 2}+C_{3} G_{3} r^{\gamma / 2}\right),
\end{aligned}
$$

where $C_{1}, C_{2}$ and $C_{3}$ are integration constants and

$$
\begin{aligned}
\gamma= & \left\{(17-8 \mathcal{B}) z^{2}-2(3 n+9-8 \mathcal{B}) z+n^{2}+6 n+1-8 \mathcal{B}\right\}^{1 / 2}, \\
F_{1}= & -2(z-1)(n-z-1) \mathcal{K}^{-1}, \\
F_{2}= & \left(\mathcal{F}_{1}-\mathcal{F}_{2}\right)\{8 z \mathcal{K}[(z-1) \mathcal{B}+2 n+z-3]\}^{-1}, \\
F_{3}= & \left(\mathcal{F}_{1}+\mathcal{F}_{2}\right)\{8 z \mathcal{K}[(z-1) \mathcal{B}+2 n+z-3]\}^{-1}, \\
G_{1}= & 2(z-1)(n+z-1) \mathcal{K}^{-1}, \\
G_{2}= & \left(\mathcal{G}_{1}+\mathcal{G}_{2}\right)\{8 z \mathcal{K}[(z-1) \mathcal{B}+2 n+z-3]\}^{-1}, \\
G_{3}= & \left(\mathcal{G}_{1}-\mathcal{G}_{2}\right)\{8 z \mathcal{K}[(z-1) \mathcal{B}+2 n+z-3]\}^{-1}, \\
\mathcal{K}= & (z-1)(n+z-1) \mathcal{B}+z(z-1)+n(n-1), \\
\mathcal{F}_{1}= & 8(z-1)[(z-1)(n+z-1) \mathcal{B}+z(z-1)+n(n-1)] \\
& \times\left[(z-1)(n+3 z-3) \mathcal{B}-2 z^{2}+(n+3) z+n(n-2)-1\right], \\
\mathcal{F}_{2}= & \gamma[n-1+(z-1) \mathcal{B}]\left\{8(1+\mathcal{B})(z-1)^{3}\right. \\
& \left.+(17 n-9+8 \mathcal{B})(z-1)^{2}+2(n+8)(n-1)(z-1)+n^{2}(n-1)-(n-1) \gamma^{2}\right\}, \\
\mathcal{G}_{1}= & 8(z-1)[2(z-1) \mathcal{B}-3 z+3 n-1][(z-1)(n+z-1) \mathcal{B}+z(z-1)+n(n-1)], \\
\mathcal{G}_{2}= & \left\{8(1+\mathcal{B})(z-1)^{3}+(17 n-9+8 \mathcal{B})(z-1)^{2}\right. \\
& \left.+2(n+8)(n-1)(z-1)+n^{2}(n-1)\right\} \gamma-(n-1) \gamma^{3} .
\end{aligned}
$$


Note that the functions $f_{1}(r), g_{1}(r)$ and $h_{1}(r)$ reduce to those given in ref. [14] for $\hat{\alpha}_{2}=\hat{\alpha}_{3}=0(\mathcal{B}=1)$ and $n=3$. For arbitrary values of $\hat{\alpha}_{2}$ and $\hat{\alpha}_{3}, \gamma$ is not an integer, and therefore the only integer power of $r$ is $r^{-n-z+1}$. However in Einstein gravity $\gamma$ can be an integer. This occurs for $z=2$ with either $n=3$ or $n=6$. For $z=2$ and $n=3, \gamma=4$ and $C_{2}=0$; the other two powers are the same and equal to -4 . Of course, the next order leading term is $r^{2(-n-z+1)}=r^{-8}$ which is a second order perturbation in $\varepsilon$. For $z=2$ and $n=6, \gamma=5$. The largest power of $r$ in the large $r$ expansion is $r^{-1}$. For other $z$ or $n$, even in Einstein gravity, the only integer power of $r$ at large $r$ for $k=0$ to first order in $\varepsilon$ (powers larger than $2(-n-z+1)$ ) is $r^{-n-z+1}$.

We next obtain the coefficients of integer powers of $r$ for $k= \pm 1$ up to the first order in $\varepsilon$. The functions $f(r), g(r)$ and $h(r)$ at large $r$ up to the first order in $\varepsilon$ may be written as

$$
\begin{aligned}
& f(r)=1+\sum_{i=1}^{2(n+z)-3} \frac{a_{i}}{r^{i}}, \\
& g(r)=1+\sum_{i=1}^{2(n+z)-3} \frac{b_{i}}{r^{i}}, \\
& h(r)=1+\sum_{i=1}^{2(n+z)-3} \frac{c_{i}}{r^{i}} .
\end{aligned}
$$

First, we consider the problem in Einstein gravity. For $n=3$ and $z=2$, all the coefficients of integer powers of $r$ up to the first order $(n \geq-7)$ are proportional to $k$ except the coefficient of $r^{-4}$. These coefficients are given in [13]. For $z=2$ and $n=6$, the first non-vanishing term is $r^{-1}$ and all the odd and even powers of $r$ are present. It is a matter of calculation to show that the coefficients of first three powers $\left(r^{-1}\right.$, $r^{-2}$ and $r^{-3}$ ) are

$$
\begin{array}{rlrl}
a_{1} & =3 c_{1}, & b_{1} & =\frac{c_{1}}{3}, \\
a_{2} & =\frac{13}{6} c_{1}^{2}+\frac{4}{5} k l^{2}, & b_{2} & =-\frac{1}{2} c_{1}^{2}+\frac{4}{5} k l^{2}, \\
a_{3} & =-\frac{1}{27} c_{1}^{3}+\frac{136}{45} k l^{2} c_{1}, b_{3}=\frac{7}{9} c_{1}^{3}+\frac{8}{15} c_{1}^{2}+\frac{4}{5} k l^{2} c_{1}, & c_{3}=\frac{29}{54} c_{1}^{3}+\frac{44}{45} k l^{2} c_{1},
\end{array}
$$

where $c_{1}$ is an arbitrary coefficient. For other values of $n$ with $z=2$ in Einstein gravity, the nonzero coefficients are those of $r^{-2}, r^{-n-z+1}, r^{-n-z-1}, \ldots r^{-n-z+1-2 m}$, where $m$ is the largest integer less than $(n+z-1) / 2$ up to the first order terms in $\varepsilon$ $(i<2(n+z-1))$. Of course, the next power of $r$ is $r^{-2 n-2 z+2}$, which is a second order term in our analysis and is nonzero even for $k=0$ case. We note that for $z=n-1$ in $(n+1)$-dimensional Einstein gravity, some logarithmic terms will appear. 
For arbitrary values of the Lovelock coefficients, all the odd powers of $1 / r$ vanish at large $r$ and the coefficients of $1 / r^{2}$ are

$$
\begin{aligned}
a_{2}= & k \frac{z l^{2}}{B}\left\{3 \hat{\alpha}_{3}\left[2 z^{3}-2(n-5) z^{2}+3(n-4)(n-5) z-\left(2 n^{2}-11 n+16\right)\right]\right. \\
& \left.-2 l^{2} \hat{\alpha}_{2}\left[z^{3}+(n-5) z^{2}+\left(n^{2}-7 n+13\right)^{2} z-\left(2 n^{2}-10 n+13\right)\right]+(n-2)[(n-3) z-2 n+5] l^{4}\right\}, \\
b_{2}= & k \frac{l^{2}}{B}\left\{3 \hat{\alpha}_{3}\left[2 z^{3}+2(n-5) z^{2}+\left(n^{2}-10 n+22\right) z-2(n-3)^{2}\right]+(n-2)[(n-4) z-2(n-3)] l^{4}\right. \\
& \left.\left.-2 l^{2} \hat{\alpha}_{2}\left[z^{3}+(n-5) z^{2}+(n-3)(n-5)\right) z-\left(2 n^{2}-11 n+15\right)\right]\right\}, \\
c_{2}= & k \frac{z l^{2}}{2 B}\left\{3 \hat{\alpha}_{3}\left[2 z^{3}+2(n-4) z^{2}+\left(n^{2}-9 n+16\right) z-2(n-2)(n-3)\right]\right. \\
& \left.-2 l^{2} \hat{\alpha}_{2}\left[z^{3}-(n-4) z^{2}+\left(n^{2}-7 n+11\right) z-\left(2 n^{2}-9 n+10\right)\right]-(n-2)[(n-3) z-2(n-2)] l^{4}\right\},
\end{aligned}
$$

where

$$
B=(z+n-3)\left\{3 \hat{\alpha}_{3}(z-2)(z+n-3)-2 \hat{\alpha}_{2} l^{2}[z(n-3)-2 n+5]-\left[z^{2}-(n-1) z+2(n-2)\right] l^{4}\right\} .
$$

The higher order coefficients with even power of $r\left(r^{-4}, r^{-6}, ..\right)$ are present and can be easily calculated but we shall not write them here as they are quite lengthy. In this case all $a_{n}$ 's are proportional to $k$. Thus, this feature is again a difference between Einstein and Lovelock gravity. That is, in Lovelock gravity all the even powers of $r$ up to $r^{-n-z+1}$ are present in the expansion of the functions at large $r$, while in Einstein gravity this does not occur for $z=2$.

\section{Acknowledgements}

This work was supported by the Natural Sciences and Engineering Research Council of Canada and Research Council of Shiraz University

\section{References}

[1] J. M. Maldacena, The large N limit of superconformal field theories and supergravity, Adv. Theor. Math. Phys. 2 (1998) 231 [Int. J. Theor. Phys. 38 (1999) 1113] [hep-th/9711200].

[2] E. Witten, Anti-de Sitter space and holography, Adv. Theor. Math. Phys. 2 (1998) 253 [arXiv:hep-th/9802150].

[3] P. Kovtun, D. T. Son, and A. O. Starinets, Viscosity in strongly interacting quantum field theories from black hole physics, Phys. Rev. Lett. 94 (2005) 111601 [hep-th/0405231].

[4] S. A. Hartnoll, Lectures on holographic methods for condensed matter physics, Class. Quant. Grav. 26 (2009) 224002 [arXiv:0903.3246 [hep-th]]. 
[5] S. A. Hartnoll, C. P. Herzog and G. T. Horowitz, Building a holographic superconductor, Phys. Rev. Lett. 101 (2008) 031601 [arXiv:0803.3295].

[6] S. A. Hartnoll and P. Kovtun, Hall conductivity from dyonic black holes, Phys. Rev. D 76 (2007) 066001 [arXiv:0704.1160].

[7] C. P. Herzog, Lectures on holographic superfluidity and superconductivity, J. Phys. A 42 (2009) 343001 [arXiv:0904.1975].

[8] T. Faulkner, H. Liu, J. McGreevy and D. Vegh, Emergent quantum criticality, Fermi surfaces and AdS2, arXiv:0907.2694.

[9] J. McGreevy, Holographic duality with a view toward many-body physics, arXiv:0909.0518.

[10] P. Koroteev and M. Libanov, On Existence of Self-Tuning Solutions in Static Braneworlds without Singularities, JHEP 0802 (2008) 104 [arXiv:0712.1136].

[11] S. Kachru, X. Liu and M. Mulligan, Gravity duals of Lifshitz-like fixed points, Phys. Rev. D 78 (2008) 106005[arXiv:0808.1725].

[12] M. Taylor, Non-relativistic holography, arXiv:0812:0530.

[13] R. B. Mann, Lifshitz topological black holes, JHEP 0906 (2009) 075 [arXiv:0905.1136].

[14] G. Bertoldi, B. A. Burrington and A. Peet, Black holes in asymptotically Lifshitz spacetimes with arbitrary critical exponent, Phys. Rev. D 80 (2009) 126003 [arXiv:09053183].

[15] K. Balasubramanian and J. McGreevy, An analytic Lifshitz black hole, Phys. Rev. D 80 (2009) 104039 [arXiv:0909.0263]].

[16] U. H. Danielsson, L. Thorlacius, Black holes in asymptotically Lifshitz spacetime, JHEP 0903 (2009) 070 [arXiv:0812.5088].

[17] E. Ayon-Beato, A. Garbarz, G. Giribet and M. Hassaine, Lifshitz black hole in three Dimensions, Phys. Rev. D 80 (2009) 104029 [arXiv:0909.1347].

[18] E. Ayon-Beato, A. Garbarz, G. Giribet and M. Hassaine, Analytic Lifshitz black holes in higher dimensions, JHEP 1004 (2010) 030 [arXiv:1001.2361].

[19] R. G. Cai, Y. Liu and Y. W. Sun, A Lifshitz black hole in four dimensional $R^{2}$ gravity, JHEP 0910 (2009) 080 [arXiv:0909.2807].

[20] D. W. Pang, On charged Lifshitz black holes, JHEP 1001 (2010) 116 [arXiv:0911.2777].

[21] M. H. Dehghani and R. Pourhasan, Thermodynamic instability of black holes of third order Lovelock gravity, Phys. Rev. D 79 (2009) 064015 [arXiv:0903.4262]. 
[22] J. de Boer, M. Kulaxizia, and A. Parnachev, Holographic Lovelock Gravities and Black Holes, JHEP 1006(2010) 008 [arXiv:0912.1877].

[23] X. O. Camanho and J. D. Edelstein, Causality in AdS/CFT and Lovelock theory, arXiv:0912.1944 [hep-th].

[24] R. C. Myers, M. F. Paulosb and A. Sinha, Holographic studies of quasi-topological gravity, arXiv:1004.2055 [hep-th].

[25] D. Lovelock, The Einstein tensor and its generalizations, J. Math. Phys. 12 (1971) 498.

[26] D. G. Boulware and S. Deser, String-generated gravity models, Phys. Rev. Lett. 55 (1985) 2656.

[27] G. Dotti, J. Oliva, and R. Troncoso, Exact solutions for the Einstein-Gauss-Bonnet theory in five dimensions: Black holes, wormholes and spacetime horns, Phys. Rev. D 76 (2007) 064038 [arXiv:0706.1830].

[28] M. H. Dehghani and M. Shamirzaie, Thermodynamics of asymptotically flat charged black holes in third order Lovelock gravity, Phys. Rev. D 72 (2005) 124015 [hep-th/0506227].

[29] V. Iyer and R. M. Wald, Some properties of Noether charge and a proposal for dynamical black hole entropy, Phys. Rev. D 50 (1994) 846 [gr-qc/9403028].

[30] S. F. Ross and O. Saremi, Holographic stress tensor for non-relativistic theories, JHEP 0909 (2009) 009 [arXiv:09071846].

[31] G. Bertoldi, B. A. Burrington and A. Peet, Thermodynamics of black branes in asymptotically Lifshitz spacetimes, Phys. Rev. D 80 (2009) 126004 [arXiv:0907.4755].

[32] J. Stachel, Globally stationary but locally static space-times: A gravitational analog of the Aharonov-Bohm effect, Phys. Rev. D 26 (1982) 1281. 